\title{
Q4 Masitinib for treatment of severely symptomatic indolent systemic mastocytosis: a randomised, placebo-controlled, phase 3 study
}

\author{
Olivier Lortholary, Marie Olivia Chandesris, Cristina Bulai Livideanu, Carle Paul, Gérard Guillet, Ewa Jassem, Marek Niedoszytko, Stéphane Barete, \\ Srdan Verstovsek, Clive Grattan, Gandhi Damaj, Danielle Canioni, Sylvie Fraitag, Ludovic Lhermitte, Sophie Georgin Lavialle, Laurent Frenzel, \\ Lawrence B Afrin, Katia Hanssens, Julie Agopian, Raphael Gaillard, Jean-Pierre Kinet, Christian Auclair, Colin Mansfield, Alain Moussy, \\ Patrice Dubrevil, Olivier Hermine
}

Lancet 2017; 389: 612-20

Published Online January 6, 2017 http://dx.doi.org/10.1016/ S0140-6736(16)31403-9

See Comment page 576

Department of Infectious Diseases and Tropical Medicine and Centre d'Infectiologie Necker-Pasteur

(Prof O Lortholary MD), Department of Hematology (M O Chandesris MD Prof O Hermine MD,

L Frenzel MD), Department of

Pathology (D Canioni MD, S Fraitag MD), INSERM U1151 and Laboratory of Onco-Hematology

(L Lhermitte MD), Institut Imagine INSERM U1163 and CNRS ERL8654 (Prof O Hermine,

L Frenzel), Centre de Référence des Mastocytoses (K Hanssens BSc, J Agopian MSc, P Dubreuil $\mathrm{PhD}$

Prof O Lortholary), Université Paris Descartes, Hôpital Necker Enfants Malades, Assistance

Publique Hôpitaux de Paris; Université Paris Descartes, Paris,

France (Prof O Lortholary); Department of Dermatology,

Mastocytosis Competence Center, Paul Sabatier University, Hôpital Larrey, Toulouse, France (C Bulai Livideanu MD, Prof ( Paul MD); Department of Dermatology, CHU Poitiers, University Hospital, Poitiers,

France (Prof G Guillet MD);

Department of Allergology, Medical University of Gdansk Gdansk, Poland (Prof Ejassem MD,

M Niedoszytko MD); Department of Dermatology and Allergology, Centre de Référence des Mastocytoses (S Barete MD) and Department of Internal Medicine, DHU I2B

(SGeorgin Lavialle MD),

Université Pierre et Marie Curie,

Hôpital Tenon, Assistance

Publique Hôpitaux de Paris,

\section{Summary}

Background Indolent systemic mastocytosis, including the subvariant of smouldering systemic mastocytosis, is a lifelong condition associated with reduced quality of life. Masitinib inhibits KIT and LYN kinases that are involved in indolent systemic mastocytosis pathogenesis. We aimed to assess safety and efficacy of masitinib versus placebo in severely symptomatic patients who were unresponsive to optimal symptomatic treatments.

Methods In this randomised, double-blind, placebo-controlled, phase 3 study, we enrolled adults (aged 18-75 years) with indolent or smouldering systemic mastocytosis, according to WHO classification or documented mastocytosis based on histological criteria, at 50 centres in 15 countries. We excluded patients with cutaneous or non-severe systemic mastocytosis after a protocol amendment. Patients were centrally randomised (1:1) to receive either oral masitinib $(6 \mathrm{mg} / \mathrm{kg}$ per day over 24 weeks with possible extension) or matched placebo with minimisation according to severe symptoms. The primary endpoint was cumulative response ( $\geq 75 \%$ improvement from baseline within weeks $8-24$ ) in at least one severe baseline symptom from the following: pruritus score of 9 or more, eight or more flushes per week, Hamilton Rating Scale for Depression of 19 or more, or Fatigue Impact Scale of 75 or more. We assessed treatment effect using repeated measures methodology for rare diseases via the generalised estimating equation model in a modified intention-to-treat population, including all participants assigned to treatment minus those who withdrew due to a non-treatment-related cause. We assessed safety in all patients who received at least one dose of study drug. This trial is registered with ClinicalTrials.gov, number NCT00814073.

Findings Between Feb 19, 2009, and July 15, 2015, 135 patients were randomly assigned to masitinib (n=71) or placebo $(n=64)$. By 24 weeks, masitinib was associated with a cumulative response of $18.7 \%$ in the primary endpoint (122.6 responses of 656.5 possible responses [weighted generalised estimating equation]) compared with $7.4 \%$ for placebo $(48.9$ of $656 \cdot 5$; difference $11.3 \%$; odds ratio $3 \cdot 6 ; 95 \%$ CI $1.2-10 \cdot 8 ; \mathrm{p}=0.0076)$. Frequent severe adverse events ( $>4 \%$ difference from placebo) were diarrhoea (eight [11\%] of 70 in the masitinib group vs one [2\%] of 63 in the placebo group), rash (four [6\%] vs none), and asthenia (four [6\%] vs one [2\%]). The most frequent serious adverse events were diarrhoea (three patients [4\%] vs one [2\%]) and urticaria (two [3\%] vs none), and no life-threatening toxicities occurred. One patient in the placebo group died (unrelated to study treatment).

Interpretation These study findings indicate that masitinib is an effective and well tolerated agent for the treatment of severely symptomatic indolent or smouldering systemic mastocytosis.

Funding AB Science (Paris, France).

\section{Introduction}

Mastocytosis is a rare disease characterised by mast cell neoplasia and aberrant mast cell activation in various tissues, leading to a heterogeneous clinical presentation and wide variety of symptoms, such as pruritus, flushes, depression, and asthenia. ${ }^{1.2}$ Although life expectancy is similar to that of the general population in the relatively indolent variants of mastocytosis-cutaneous mastocytosis and indolent systemic mastocytosis, including the subvariant of smouldering systemic mastocytosisabout a third of patients will experience severe symptoms of mast cell mediator release. A greatly increased occurrence and severity of such symptoms is reported in systemic mastocytosis when compared with cutaneous mastocytosis. ${ }^{1,2}$ Treatment decisions are based on the presence of bone marrow mast cell infiltration and severity of symptoms, with the main objective being a sustained improvement of symptoms-ie, inhibition of mast cell mediator release.

Genetic aberrations are known to be involved in the pathogenesis of systemic mastocytosis, predominantly the KIT Asp816Val (D816V) mutation, with an emerging understanding that a wide variety of other KIT mutations and mast cell regulatory genes might also be implicated. ${ }^{3.4}$ Indeed, type and severity of symptoms are independent of KIT Asp816Val status. ${ }^{1,5,6}$ Masitinib is an oral tyrosine 
Research in context

\section{Evidence before this study}

We searched PubMed without date restriction for reports pertaining to phase 2 or 3 clinical trials in indolent systemic mastocytosis using the search terms of "systemic mastocytosis" [All Fields] filtered for "Clinical Trial, Phase II" and "Clinical Trial, Phase III" [publication type]. We did not apply any language restrictions, but used search terms in English only. We identified 11 previous clinical trials matching these search criteria, describing seven potential treatments for indolent systemic mastocytosis. No trial focused solely on indolent systemic mastocytosis, with this cohort representing a subgroup of overall populations that also comprised advanced systemic mastocytosis or cutaneous mastocytosis patients. All trials were open label, non-comparative (singlearm), phase 2 studies evaluating various compounds including masitinib, dasatinib, everolimus, imatinib, interferon alfa, nilotinib, and thalidomide. Results were mixed, varying from potential therapeutic benefit in select patients to no appreciable clinical efficacy.

\section{Added value of this study}

To our knowledge, this is the first phase 3 prospective, randomised placebo-controlled study of a treatment for indolent systemic mastocytosis. We show a significant and clinically meaningful treatment benefit in this difficult-to-treat population, with a demonstrated possibility of effective long-term management.

\section{Implications of all the available evidence}

The observed positive benefit-risk ratio supports the use of masitinib for patients with severely symptomatic indolent systemic mastocytosis. Masitinib might be a new treatment option for adult patients with severely symptomatic indolent systemic mastocytosis, including those with the subvariant of smouldering systemic mastocytosis, who are unresponsive to existing symptomatic treatments. Statistical design features of the study and mechanistic implications of targeting of non-clonal mast cells or KIT Asp816Val-independent signalling pathways could influence future trial design in mastocytosis.

kinase inhibitor that targets wild-type KIT (50\% inhibitory concentration $\left[\mathrm{IC}_{50}\right] 200 \mathrm{nM}$ ) with greater potency in vitro than KIT Asp816Val $\left(\mathrm{IC}_{50} 5.0 \mu \mathrm{M}\right)$, as well as targeting LYN and FYN at submicromolar concentrations.? Wildtype KIT, LYN, and FYN have crucial roles in the survival and function of mast cells, including mediator release. ${ }^{8,9}$ Masitinib has a higher selectivity for these targeted kinases than other KIT inhibitors, which is likely to lower the risk of off-target toxicities. ${ }^{10,11}$ Phase 2 study results have shown its potential efficacy in patients with indolent forms of mastocytosis, regardless of their KIT Asp816Val status. ${ }^{12}$

We aimed to assess the safety and efficacy of masitinib for treatment of indolent forms of systemic mastocytosis in patients who were unresponsive to optimal symptomatic treatment.

\section{Methods}

\section{Study design and participants}

This multicentre, parallel-group, randomised, doubleblind, placebo-controlled, phase 3 study (AB06006) was initiated in 2009 and conducted in 15 countries (Austria, Czech Republic, France, Germany, Greece, India, Italy, Latvia, Poland, Russia, Slovakia, Spain, Switzerland, UK, and USA) across 50 active centres.

The study protocol and amendment were approved by the relevant institutional review boards at individual enrolment centres or ethics committees and conducted according to the Declaration of Helsinki. All patients provided written informed consent.

Eligible patients were aged 18-75 years and had indolent or smouldering systemic mastocytosis according to the WHO classification, ${ }^{13-15}$ or documented mastocytosis based on histological criteria of typical mast cell infiltrates in a multifocal or diffuse pattern in skin or bone marrow biopsy. The latter criterion encompasses all patients satisfying the WHO classification but also selects those patients matching inclusion criteria from the masitinib phase 2 trials and AFIRMM survey. ${ }^{1,2}$ Consequently, these inclusion criteria are broader than the WHO classification. To ensure consistency in the investigators' application of diagnostic criteria, a blinded central document reading was used to verify patient eligibility for inclusion to the intention-to-treat (ITT) population based on a set of unifying criteria that encompassed the WHO classification (appendix p 2). We did primary analysis on the ITT population as defined via this central document review. Additional eligibility criteria included severe symptoms of mast cell mediator release at baseline-pruritus score of 9 or more determined via a patient perception questionnaire, ${ }^{1}$ at least eight flushes per week, Hamilton Rating Scale for Depression score of 19 or more, ${ }^{16}$ or Fatigue Impact Scale total score of 75 or more ${ }^{17}$-and documented failure of at least one symptomatic treatment used at optimal dose-eg, H1-antihistamines, H2-antihistamines, proton pump inhibitors, sodium cromoglicate, antidepressants, and leukotriene antagonists (appendix p 12). Patients were ineligible if presenting with one of the following variants of mastocytosis: cutaneous mastocytosis (as per the amended protocol version 6.0), undocumented indolent systemic mastocytosis or smouldering systemic mastocytosis, systemic mastocytosis with an associated clonal haematological non-mast-celllineage disease, mast cell leukaemia, or aggressive systemic mastocytosis; patients presenting with inadequate organ function defined via blood test levels; vulnerable populations such as patients with life expectancy of less than 6 months, known diagnosis of human
Paris, France; Hanns A Pielenz Clinical Research Center for Myeloproliferative Neoplasms, Department of Leukemia, MD Anderson Cancer Center, Houston, TX, USA

(Prof S Verstovsek MD); Department of Dermatology, Norfolk \& Norwich University Hospital, Norwich, UK (C Grattan MD); Department of Haematology, University Hospital of Caen, Institut d'Hématologie de Basse Normandie, School of Medicine, University of Lower Normandy, Caen, France (Prof G Damaj MD); Division of Hematology, Oncology \& Transplantation University of Minnesota, Minneapolis, MN, USA (L B Afrin MD); INSERM U1068, CRCM (Signaling,

Hematopoiesis and Mechanism of Oncogenesis), Institut Paoli-Calmettes, Aix-Marseille Université, CNRS, UMR7258, Marseille, France (P Dubreuil); Human Histopathology and Animal Models, Infection and Epidemiology Department, Institut Pasteur; Université Paris Descartes; Centre Hospitalier Sainte-Anne, Paris, France (Prof R Gaillard MD); Department of Pathology Harvard Medical School and Beth Israel Deaconess Medical Center, Boston, MA, USA (Prof J-P Kinet MD); Laboratoire de Biologie et Pharmacologie appliqué, CNRS UMR 8113, Ecole Normale Supérieure de Cachan, Université Paris Saclay Paris, France (C Auclair PhD); $A B$ Science, Paris, France

(C Mansfield PhD, A Moussy MBA P Dubreuil, Prof O Hermine, ( Auclair, K Hanssens, J Agopian); and INSERM, La Ligue Nationale Contre le Cancer (équipe labelliseé), Paris, France (P Dubreuil)

Correspondence to: Prof Olivier Hermine, Université Sorbonne Paris Cité, Department of Clinical Hematology, National Center of Mastocytosis, Hôpital Necker, 75015 Paris, France ohermine@gmail.com

For the statistical analysis plan, study protocol, and summary of protocol changes see http:// www.ab-science.com/pdf/ Lortholary_et_al_Lancet Protocol_online.pdf

See Online for appendix 
immunodeficiency virus (HIV) infection, known cardiac disorders, or Eastern Cooperative Oncology Group performance status greater than 2; previous treatment with any tyrosine kinase inhibitor or treatment with any investigational agent within 4 weeks prior to baseline; and change in the symptomatic treatment of mastocytosis or administration of any new treatment of mastocytosis within 4 weeks prior to baseline (appendix p 7).

\section{Randomisation and masking}

Patients were centrally randomised to masitinib or placebo in a 1:1 ratio using an interactive voice response system and minimisation method according to the covariates of pruritus score, number of flushes per week, depression (measured by the Hamilton Rating Scale for Depression), asthenia (measured by the Fatigue Impact Scale), and country. Masitinib and placebo capsules were identical except for the active ingredient and both produced by Excella GmbH (Feucht, Germany), with no difference in dispensing of medication. The investigators, patients, data analysts, and the trial funder were blinded to the randomisation sequence and treatment assignment.

\section{Procedures}

Masitinib was administered orally at $6 \mathrm{mg} / \mathrm{kg}$ per day in two daily doses over 24 weeks with a possibility of a double-blind extension period. Long-term analysis was done over the timeframe of weeks 8-96. Patients were assessed at weeks $8,12,16,20$, and 24 during the 24-week treatment period, with assessments every 12 weeks thereafter if entering the 2-year (96-week) extension period. In the event of severe toxicity related to masitinib, treatment interruption or dose reduction was permitted according to predefined criteria (appendix p 10). Protocol amendments were implemented between 3.5 years and 2 years prior to database unmasking, owing to an emergent risk of masitinib-related severe neutropenia and severe skin toxicity. Protocol amendment version 6.0 aimed to modify the protocol's benefit-risk balance to identify the patient population with greatest medical need. The amendment introduced four key changes: enrolment was restricted to patients with severe baseline symptoms of mast cell mediator release; enrolment was restricted to indolent systemic mastocytosis, including the subvariant of smouldering systemic mastocytosis, because these patients exhibit greater symptom severity than do those with cutaneous mastocytosis; ${ }^{1}$ the threshold for positive treatment response was increased from $50 \%$ to $75 \%$, thereby enhancing the clinical relevance of improvement; and, as recommended in the European Medicines Agency (EMA) guidelines for clinical trial design for rare diseases, the treatment effect was tested using a repeated measures methodology-namely, longitudinal analysis with respect to symptoms as opposed to patient response rate at a single point in time. ${ }^{18}$ Patients with severely symptomatic systemic mastocytosis were defined as those with at least one severe baseline symptom of mast cell mediator release. Thus, only patients with indolent systemic mastocytosis meeting the prospectively declared inclusion criteria specified in this amendment were included for final analysis-ie, the ITT population.

Administration of concomitant optimal symptomatic treatments was allowed (appendix p 12); however, administration of any other kinase inhibitor, interferon alfa, or cladribine was not permitted during the study period.

\section{Outcomes}

The prospectively declared primary endpoint (referred to hereafter as $4 \mathrm{R} 75 \%$ ) was cumulative response in at least one of four severe baseline symptoms of mast cell mediator release (pruritus, flushes, depression, or asthenia). We defined response as a $75 \%$ improvement from baseline for any of these four symptoms. We defined cumulative response as the number of actual responses between weeks 8 and 24, divided by the total number of possible responses over the same treatment period (ie, with five scheduled visits, each patient had a maximum of five to 20 possible responses depending on the number of severe baseline symptoms).

Secondary endpoints were cumulative response in at least one of three severe baseline symptoms of mast cell mediator release (pruritus, flushes, or depression) with response defined as an improvement of at least $75 \%$ from baseline for any of these three symptoms (referred to hereafter as $3 \mathrm{R} 75 \%$ ); cumulative response in pruritus or flushes with response defined as an improvement of at least $75 \%$ from baseline for either symptom (2R75\%); cumulative response in pruritus alone; improvement of urticaria pigmentosa as measured via cumulative change in affected body surface area relative to baseline; disappearance of Darier's sign; mean change of tryptase level at week 24 relative to baseline in patients with baseline tryptase level greater than $20 \mu \mathrm{g} / \mathrm{L}$; cumulative response in micturition and stool frequency among patients with a baseline of eight or more per day and four or more per day, respectively; and quality-of-life measures such as the AFIRMM questionnaire (version 2). The safety profile of masitinib was compared with placebo according to occurrence and severity of adverse events, regardless of causality.

\section{Statistical analysis}

For the primary efficacy analysis, a cumulative total of 1065 possible response evaluations was required to detect a difference of $12.5 \%$ in $4 \mathrm{R} 75 \%$ between treatment arms (based on the assumption of an average 1.5 severe baseline symptoms per patient, over five assessment timepoints for 142 patients, and a response of $21.0 \%$ in the masitinib arm vs $8.5 \%$ in the placebo arm) with a power of $80 \%$ and significance level of 0.05 (two-sided log-rank test). The hypothesised response estimates were based on empirical knowledge from phase 2 data. 


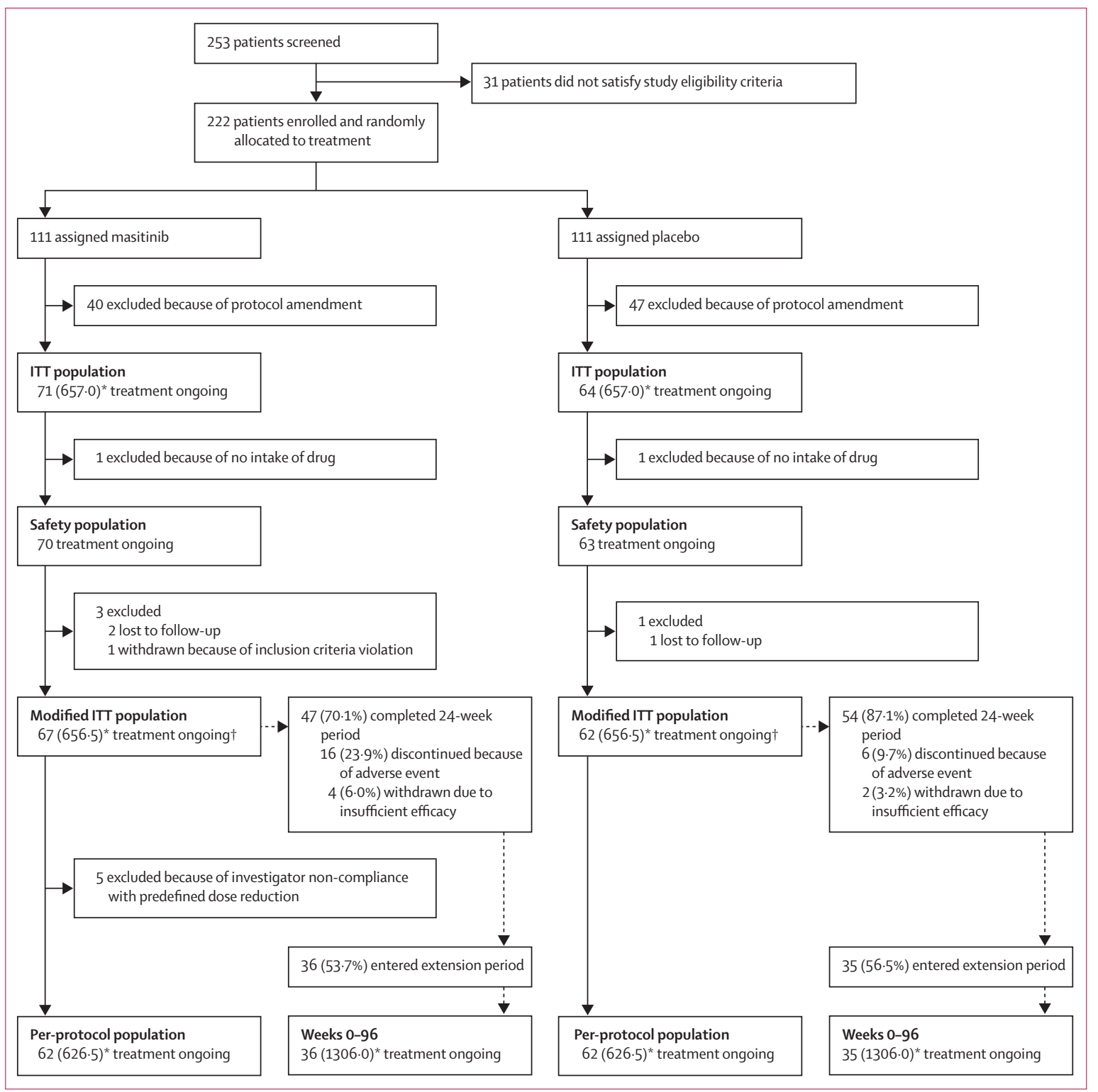

Figure: Trial profile

A study amendment (August, 2013, as per protocol version 6.0) restricted enrolment to patients with severe indolent and smouldering systemic mastocytosis. Consequently, 87 patients with cutaneous mastocytosis or non-severe systemic mastocytosis recruited prior to this amendment were excluded from the ITT population. A weighted GEE model was used to provide total possible cumulative responses assessable in calculation of study endpoints. ITT and per-protocol populations represent sensitivity tests of primary analysis. ITT=intention-to-treat. GEE=generalised estimating equation. *Total possible cumulative responses assessable in calculation of the 4 R75\% endpoint according to GEE model. †Primary endpoint corresponds to modified ITT population.

We did primary efficacy analysis according to a modified ITT population (ITT population minus those withdrawing for a well documented, non-treatmentrelated cause-eg, no intake of drug), with results verified via analysis on the ITT population (all eligible patients assigned to treatment, irrespective of actual treatment received), as well as other sensitivity analyses including the per-protocol population (modified ITT minus those with a major protocol deviation) and modified ITT observed cases dataset (see appendix p 2).
The safety population comprised all ITT patients who received at least one dose of study medication. All main, sensitivity, and subgroup analyses reported here were prespecified in the study's statistical analysis plan prior to unblinding, and we did no interim analyses. We considered missing data as failure for primary and secondary analyses, did sensitivity analyses with the last observation carried forward or observed cases approach, and obtained the statistical test $\mathrm{p}$ value for the primary analysis via a re-randomisation (10000 replicate) test. 


\begin{tabular}{|c|c|c|}
\hline & Masitinib $(n=67)$ & Placebo $(n=62)$ \\
\hline \multicolumn{3}{|l|}{ Demographic } \\
\hline Age (years) & $45 \cdot 3(11 \cdot 1 ; 19-69)$ & $49 \cdot 2(12 \cdot 7 ; 27-86)$ \\
\hline \multicolumn{3}{|l|}{ Sex } \\
\hline Female & $50 / 67(75 \%)$ & $41 / 62(66 \%)$ \\
\hline Male & $17 / 67(25 \%)$ & $21 / 62(34 \%)$ \\
\hline \multicolumn{3}{|l|}{ c-KIT status } \\
\hline Clonal (KIT Asp816Val) & $63 / 67(94 \%)$ & $53 / 62(86 \%)$ \\
\hline KIT wild-type & $1 / 67(2 \%)$ & $7 / 62(11 \%)$ \\
\hline Unknown & $3 / 67(5 \%)$ & $2 / 62(3 \%)$ \\
\hline \multicolumn{3}{|l|}{ Disease type } \\
\hline $\begin{array}{l}\text { Indolent systemic } \\
\text { mastocytosis* }\end{array}$ & $54 / 67(81 \%)$ & $49 / 62(79 \%)$ \\
\hline $\begin{array}{l}\text { Smouldering systemic } \\
\text { mastocytosis* }\end{array}$ & $6 / 67(9 \%)$ & $7 / 62(11 \%)$ \\
\hline Unclassified & $7 / 67(10 \%)$ & $6 / 62(10 \%)$ \\
\hline \multicolumn{3}{|c|}{ Severe symptoms at baseline } \\
\hline \multicolumn{3}{|l|}{ Pruritus } \\
\hline Cases (\%) & $45 / 67(67 \%)$ & $42 / 62(68 \%)$ \\
\hline Mean score (SD) & $9 \cdot 0(3 \cdot 0)$ & $9 \cdot 1(3 \cdot 6)$ \\
\hline \multicolumn{3}{|l|}{ Flushes } \\
\hline Cases (\%) & $18 / 66(27 \%)$ & $17 / 62(27 \%)$ \\
\hline Mean (SD) & $8.0(9.6)$ & $6 \cdot 4(7 \cdot 4)$ \\
\hline \multicolumn{3}{|l|}{ Depression (HAMD-17) } \\
\hline Cases (\%) & $23 / 67(34 \%)$ & $27 / 62(44 \%)$ \\
\hline Mean score (SD) & $16.0(7 \cdot 4)$ & $17 \cdot 3(8 \cdot 1)$ \\
\hline \multicolumn{3}{|l|}{ Asthenia (FIS) } \\
\hline Cases (\%) & $50 / 66(76 \%)$ & $46 / 61(75 \%)$ \\
\hline Mean score (SD) & $90 \cdot 2(37 \cdot 1)$ & $89 \cdot 4(34 \cdot 3)$ \\
\hline \multicolumn{3}{|c|}{ Objective marker of mast cell activation } \\
\hline \multicolumn{3}{|l|}{ Tryptase level (>20 $\mu \mathrm{g} / \mathrm{L})$} \\
\hline Number of cases (\%) & $46 / 60(77 \%)$ & $44 / 62(80 \%)$ \\
\hline Mean (SD) & $75 \cdot 8(120)$ & $72 \cdot 2(75 \cdot 6)$ \\
\hline BSA urticaria pigmentosa $\dagger$ & $87.8(48.0)$ & $101 \cdot 0(46 \cdot 3)$ \\
\hline Darier's sign & $21 / 25(84 \%)$ & $19 / 27(70 \%)$ \\
\hline \multicolumn{3}{|c|}{$\begin{array}{l}\text { Data are mean (SD; range) or n/number assessed (\%) unless otherwise stated. } \\
\text { HAMD-17=Hamilton Rating Scale for Depression. FIS=Fatigue Impact Scale. } \\
\text { BSA=body surface area score corrected with Wallace formula. }{ }^{*} \text { Classification } \\
\text { according to investigator. }+ \text { Systemic mastocytosis patients presenting with } \\
\text { mastocytosis in the skin, specifically urticaria pigmentosa. }\end{array}$} \\
\hline
\end{tabular}

We calculated the difference between treatment arms using the generalised estimating equation (GEE) approach (logit-link function) with treatment, symptom (pruritus, flushes, depression, and asthenia), and assessment schedule (weeks 8, 12, 16, 20, and 24) included as parameters in the model. ${ }^{19}$ This approach simultaneously tests for effect in all four outcome measures specified in the primary endpoint, taking into account correlation across variables and across time so that valid inferences can be assured.

This trial is registered with ClinicalTrials.gov, number NCT00814073.

\section{Role of the funding source}

The funder (AB Science; Paris, France) was involved in the study design; data collection, analysis, and interpretation; and manuscript preparation and submission. OH, OL, and AM had full access to all the data in the study and final responsibility to submit for publication.

\section{Results}

Between Feb 19, 2009, and July 15, 2015, 253 patients were screened, from which 222 were randomised to masitinib $(n=111)$ or placebo $(n=111)$. A protocol amendment in August, 2013, restricting enrolment to severe systemic mastocytosis resulted in exclusion of 87 patients (40 from the masitinib arm, 47 from the placebo arm). As a result, the prospectively declared ITT population consisted of 135 patients (71 masitinib, 64 placebo), 108 (80\%) of whom satisfied the WHO classification $^{13,15}$ for systemic mastocytosis. The safety $(n=133)$, modified ITT $(n=129)$, and per-protocol $(n=124)$ populations were defined from the ITT population (figure, appendix p 2). Notably, the ITT and modified ITT populations were almost identical for efficacy assessment because no patient excluded from the modified ITT population had data beyond week 8 , which was the first timepoint included for cumulative data analysis Database lock was on Nov 24, 2015.

Baseline characteristics for the modified ITT population are provided in table 1 , and were similar for the ITT, per-protocol, and safety populations (data not shown). Mean exposure to masitinib in the modified ITT population over the study duration was 18.9 (SD 22.0) months (range $0 \cdot 1-74 \cdot 1$ ) versus $16.4(19 \cdot 3)$ months $(0 \cdot 7-72 \cdot 3)$ for placebo.

268 severe symptoms (pruritus, flushes, depression, and asthenia) were recorded at baseline in the modified IT'T population (136 in the masitinib group vs 132 in the placebo group). This number corresponds to a cumulative total of 1340 possible response evaluations for the primary analysis, indicating that the study was sufficiently powered. At baseline, severe pruritus was reported in about two thirds of patients from both treatment arms, severe flushes in about 27\%, severe depression in about $39 \%$, and severe asthenia in about $75 \%$ (table 1).

At 24 weeks of treatment, masitinib was associated with a $4 \mathrm{R} 75 \%$ of $18.7 \%$ versus $7.4 \%$ for placebo (odds ratio [OR] 3.6; 95\% CI 1.2-10 - 8, $\mathrm{p}=0 \cdot 0076$; table 2). This positive outcome was verified in the ITT population, as well as all predefined sensitivity analyses on the primary endpoint. Subgroup analysis in patients with KIT Asp816Val showed a significant response in favour of masitinib, with a $4 \mathrm{R} 75 \%$ of $20.2 \%(117.6$ of 581.5$)$ for masitinib versus $7.4 \%(42.8$ of 581.5$)$ for placebo $(4 \cdot 5 ; 1 \cdot 1-17 \cdot 8, \mathrm{p}=0 \cdot 0316)$ (appendix p 16). Subgroup analysis for other KIT cohorts was not possible owing to the small number of these patients. 


\begin{tabular}{|c|c|c|c|c|c|}
\hline & Masitinib & Placebo & Difference & Odds ratio $(95 \% \mathrm{Cl})$ & p value \\
\hline \multicolumn{6}{|l|}{ Primary outcome } \\
\hline \multicolumn{6}{|l|}{$4 \mathrm{R} 75 \%$} \\
\hline Modified ITT (MDF) & $122 \cdot 6 / 656 \cdot 5(19 \%)$ & $48.9 / 656 \cdot 5(7 \%)$ & $11 \cdot 2 \%$ & $3 \cdot 6(1 \cdot 2-10 \cdot 8)$ & $0.0076^{*}$ \\
\hline ITT & $122 \cdot 7 / 657 \cdot 0(19 \%)$ & $49 \cdot 8 / 657 \cdot 0(8 \%)$ & $11 \cdot 1 \%$ & $3 \cdot 3(1 \cdot 2-9 \cdot 1)$ & 0.0102 \\
\hline Modified ITT (OC) & $136 \cdot 4 / 565 \cdot 0(24 \%)$ & $44 \cdot 4 / 565 \cdot 0(8 \%)$ & $16 \cdot 3 \%$ & $4 \cdot 9(1 \cdot 6-15 \cdot 1)$ & 0.0014 \\
\hline Per-protocol & $125 \cdot 8 / 626 \cdot 5(20 \%)$ & $46 \cdot 5 / 626 \cdot 5(7 \%)$ & $12 \cdot 7 \%$ & $3 \cdot 9(1 \cdot 3-11 \cdot 5)$ & 0.0048 \\
\hline \multicolumn{6}{|l|}{ Secondary outcomes } \\
\hline \multicolumn{6}{|l|}{$3 R 75 \%$} \\
\hline Modified ITT (MDF) & $104 \cdot 5 / 423 \cdot 5(25 \%)$ & $41 \cdot 5 / 423 \cdot 5(10 \%)$ & $14.9 \%$ & $3 \cdot 1(1 \cdot 4-6 \cdot 9)$ & 0.0071 \\
\hline Modified ITT (OC) & $117 \cdot 2 / 362 \cdot 0(32 \%)$ & $37.5 / 362 \cdot 0(10 \%)$ & $22.0 \%$ & $4 \cdot 1(1 \cdot 8-9 \cdot 1)$ & 0.0008 \\
\hline Per-protocol & $107 \cdot 0 / 403 \cdot 5(27 \%)$ & $39 \cdot 4 / 403 \cdot 5(10 \%)$ & $16 \cdot 8 \%$ & $3.3(1.5-7.5)$ & 0.0038 \\
\hline \multicolumn{6}{|l|}{$2 \mathrm{R} 75 \%$} \\
\hline Modified ITT (MDF) & $81.9 / 301 \cdot 5(27 \%)$ & $32 \cdot 2 / 301 \cdot 5(11 \%)$ & $16 \cdot 5 \%$ & $2 \cdot 6(1.1-6.6)$ & 0.0380 \\
\hline Modified ITT (OC) & $92 \cdot 2 / 254 \cdot 5(36 \%)$ & $28 \cdot 8 / 254 \cdot 5(11 \%)$ & $24 \cdot 9 \%$ & $3 \cdot 8(1.5-9 \cdot 4)$ & 0.0042 \\
\hline Per-protocol & $83 \cdot 7 / 284 \cdot 0(29 \%)$ & $30 \cdot 3 / 284 \cdot 0(11 \%)$ & $18 \cdot 8 \%$ & $2 \cdot 9(1 \cdot 2-7 \cdot 2)$ & 0.0220 \\
\hline \multicolumn{6}{|l|}{ Pruritus } \\
\hline Modified ITT (MDF) & $47 \cdot 1 / 214 \cdot 5(22 \%)$ & $15 \cdot 6 / 214 \cdot 5(7 \%)$ & $14 \cdot 7 \%$ & $3 \cdot 1(1 \cdot 1-8 \cdot 9)$ & 0.0322 \\
\hline Modified ITT (OC) & $53 \cdot 0 / 178 \cdot 5(30 \%)$ & $14.0 / 178.5(8 \%)$ & $21.8 \%$ & $4 \cdot 2(1 \cdot 5-12 \cdot 0)$ & 0.0071 \\
\hline Per-protocol & $50 \cdot 0 / 202 \cdot 0(25 \%)$ & $14 \cdot 7 / 202 \cdot 0(7 \%)$ & $17 \cdot 5 \%$ & $3 \cdot 7(1 \cdot 3-10 \cdot 5)$ & 0.0146 \\
\hline \multicolumn{6}{|l|}{ Flushes } \\
\hline Modified ITT (MDF) & $34 \cdot 7 / 87 \cdot 0(40 \%)$ & $16 \cdot 6 / 87 \cdot 0(19 \%)$ & $20.8 \%$ & $3 \cdot 0(0 \cdot 9-10 \cdot 7)$ & 0.09 \\
\hline Modified ITT (OC) & $39 \cdot 3 / 76 \cdot 0(52 \%)$ & $14 \cdot 8 / 76 \cdot 0(20 \%)$ & $33 \cdot 3 \%$ & $3 \cdot 8(1 \cdot 1-13 \cdot 8)$ & 0.0402 \\
\hline Per-protocol & $33 \cdot 8 / 82 \cdot 0(41 \%)$ & $15 \cdot 6 / 82 \cdot 0(19 \%)$ & $22 \cdot 2 \%$ & $3 \cdot 1(0 \cdot 9-10 \cdot 9)$ & 0.09 \\
\hline \multicolumn{6}{|l|}{ HAMD-17 } \\
\hline Modified ITT (MDF) & $22 \cdot 7 / 122 \cdot 0(19 \%)$ & $9 \cdot 3 / 122 \cdot 0(8 \%)$ & $11.0 \%$ & $2 \cdot 7(0 \cdot 7-11 \cdot 1)$ & 0.17 \\
\hline Modified ITT (OC) & $24 \cdot 9 / 107 \cdot 5(23 \%)$ & $8 \cdot 7 / 107 \cdot 5(8 \cdot 1 \%)$ & $15.0 \%$ & $3 \cdot 3(0 \cdot 8-13 \cdot 8)$ & 0.10 \\
\hline Per-protocol & $23 \cdot 2 / 119 \cdot 5(19 \%)$ & $9 \cdot 1 / 119 \cdot 5(8 \%)$ & $11.8 \%$ & $2.9(0.7-11.8)$ & 0.14 \\
\hline \multicolumn{6}{|l|}{ FIS } \\
\hline Modified ITT (MDF) & $18 \cdot 1 / 233 \cdot 0(8 \%)$ & $7 \cdot 4 / 233 \cdot 0(3 \%)$ & $4 \cdot 6 \%$ & $4 \cdot 8(1 \cdot 0-23 \cdot 4)$ & 0.0499 \\
\hline Modified ITT (OC) & $19 \cdot 3 / 203 \cdot 0(10 \%)$ & $6.9 / 203.0(3 \%)$ & $6 \cdot 1 \%$ & $7 \cdot 0(1 \cdot 1-46 \cdot 3)$ & 0.0448 \\
\hline Per-protocol & $18.8 / 223.0(8 \%)$ & $7 \cdot 1 / 223 \cdot 0(3 \%)$ & $5 \cdot 2 \%$ & $5 \cdot 5(1 \cdot 1-27 \cdot 4)$ & 0.0370 \\
\hline \multicolumn{6}{|c|}{$\begin{array}{l}\text { Cumulative response based on generalised estimating equation (GEE) model, with missing data considered as failure (MDF), unless stated otherwise. Response rates } \\
\text { expressed as weighted ratio of sum of actual responses between weeks } 8 \text { and } 24 \text { divided by the total number of possible responses over the same treatment period. Primary } \\
\text { and secondary analyses were based on the modified ITT (MDF) dataset. Sensitivity analyses were based on the ITT, modified ITT (OC), and per-protocol datasets. } \\
4 R 75 \%=\text { cumulative response in at least one of four severe baseline symptoms (pruritus, flushes, depression, or asthenia). ITT=intention-to-treat population. OC=observed } \\
\text { cases dataset. 3R75\%=cumulative response in at least one of three severe baseline symptoms (pruritus, flushes, or depression). } 2 R 75 \%=\text { =cumulative response in at least one of } \\
\text { two severe baseline symptoms (pruritus or flushes). HAMD-17=Hamilton Rating Scale for Depression. FIS=Fatigue Impact Scale. *Based on re-randomisation. }\end{array}$} \\
\hline
\end{tabular}

We also noted improvement in secondary endpoints with masitinib (table 2). Endpoints representative of objective markers of mast cell activation were also positive (appendix p 11). At week 24, the mean change of tryptase level from baseline in the modified ITT population was a decrease of $18.0 \%$ in the masitinib arm versus an increase of $2 \cdot 2 \%$ in the placebo arman absolute difference of $20.2 \% \quad(\mathrm{p}<0.0001)$. The response of urticaria pigmentosa lesions to masitinib differed when compared with placebo $(p=0 \cdot 0210)$ as evidenced by a decrease in average body surface area of $12.3 \%$ for masitinib versus an increase of $15.9 \%$ for placebo-an absolute difference of $28.2 \%$. The response to masitinib included one KIT Asp816Valpositive patient who had a complete response at week
24 (from baseline body surface area of 18\%). This observation was supported by abolition of Darier's sign in $18.9 \%$ of patients treated with masitinib versus $2.7 \%$ treated with placebo-an absolute difference of $16 \cdot 2 \%(\mathrm{p}=0 \cdot 0187$; appendix $\mathrm{p} 11)$.

Among patients entering the extension period, of whom 36 received masitinib treatment and 35 received placebo, a sustained response was observed in the masitinib group when compared with placebo for primary, secondary, and sensitivity outcomes (appendix p 11).

Table 3 shows a summary of safety results during the 24-week treatment period, regardless of causality. The most frequently occurring severe adverse events were diarrhoea (eight [11\%] of 70 in the masitinib group vs one 
[2\%] of 63 in the placebo group), rash (four [6\%] vs none), asthenia (four [6\%] vs one [2\%]), peripheral oedema (two [3\%] vs none), pruritus (three [4\%] vs one [2\%]), and neutropenia (three [4\%] vs one [2\%]; appendix p 13). The most frequent serious adverse events were diarrhoea (three patients [4\%] vs one [2\%]) and urticaria (two [3\%] vs none; appendix p 13). No deaths were reported in the masitinib group, whereas one death, unrelated to study treatment, was reported in the placebo group. Overall, more adverse events occurred during the first 6 months in the masitinib group than in the placebo group (table 3 , appendix $\mathrm{p}$ 13).

Long-term safety over the extension period was assessed according to incidence per patient-months of exposure; this measure is more appropriate than frequency of adverse events given that some patients had been exposed to masitinib for over 2 years. This analysis revealed a comparable incidence of severe and serious adverse events between masitinib and placebo (table 4).

\section{Discussion}

Treatment with masitinib resulted in a therapeutic benefit across a diverse range of symptoms in patients with severely symptomatic indolent systemic mastocytosis who were unresponsive to optimal symptomatic treatments. Moreover, the response criterion of greater than $75 \%$ improvement in at least one severe baseline symptom constitutes a clinically meaningful effect, as evidenced by comparison with published recommendations on response evaluation..$^{20}$ Data from the extension period showed that masitinib can maintain remission of symptoms for over 2 years; this is supported by results of the GEE model-a powerful tool for making statistical inference on longitudinal data. ${ }^{21}$ This observation is important, given that indolent systemic mastocytosis is a chronic condition that requires lifelong management.

The primary analysis was supported by improvements seen in the predefined sensitivity analyses, notably the ITT population, and secondary analyses relating to patientreported symptomatic endpoints, as well as objective

\begin{tabular}{|c|c|c|c|}
\hline & Masitinib $(n=70)$ & Placebo $(n=63)$ & Difference \\
\hline At least one adverse event & $70(100 \%)$ & $63(100 \%)$ & $0.0 \%$ \\
\hline Death & 0 & $1(2 \%)$ & $-1 \cdot 6 \%$ \\
\hline Non-fatal serious adverse event & $20(29 \%)$ & $12(19 \%)$ & $9 \cdot 5 \%$ \\
\hline Severe adverse event & $35(50 \%)$ & $22(35 \%)$ & $15 \cdot 1 \%$ \\
\hline $\begin{array}{l}\text { Adverse event leading to permanent discontinuation } \\
\text { (except death) }\end{array}$ & $17(24 \%)$ & $4(6 \%)$ & $17 \cdot 9 \%$ \\
\hline In per-protocol population & $12 / 62(19 \%)$ & $4 / 62(6 \%)$ & $12 \cdot 9 \%$ \\
\hline Adverse event leading to study treatment dose reduction & $15(21 \%)$ & $1(2 \%)$ & $19.8 \%$ \\
\hline \multicolumn{4}{|c|}{$\begin{array}{l}\text { Data are number of patients (\%) affected. All data refer to safety population unless otherwise stated. For the } \\
\text { per-protocol masitinib arm, five patients were excluded owing to investigator non-compliance to predefined protocol } \\
\text { safety rules regarding dose reduction. Adverse events reported according to any causality. Adverse event intensity } \\
\text { count is cumulative. }\end{array}$} \\
\hline
\end{tabular}

endpoints representative of mast cell activation (tryptase, Darier's sign, and urticaria pigmentosa). Depression and asthenia are potential psychiatric manifestations of mast cell activation and can have a negative influence on the wellbeing of patients with systemic mastocytosis. ${ }^{1,2,22,23}$ Thus, the improvement in endpoints, such as the Fatigue Impact Scale and Hamilton Rating Scale for Depression (non-significant), and their associated $4 \mathrm{R} 75 \%$ and $3 \mathrm{R} 75 \%$ composite endpoints is indicative that masitinib can positively affect neuropsychiatric manifestations of systemic mastocytosis. These efficacy data confirm observations from related phase 2 studies (appendix p 20)..$^{24}$ A common issue for clinical trials in orphan diseases is low sample number. In this study, we circumvented this problem by using the EMA-recommended repeated measures methodology ${ }^{18}$ Despite the odds ratio confidence intervals for primary and secondary endpoints being wide, a lower boundary of at least unity supports the superiority of masitinib over placebo. Use of a blinded central document reading introduced a risk of post-randomisation imbalance that was managed via weighting of each observation (appendix p 2). Sensitivity analyses that omitted the weighting function showed this process introduced negligible bias with closely matched data to the primary analysis (data not shown). Another complication for data interpretation arises because of the definition of indolent systemic mastocytosis used in this study is broader than the WHO classification. Among the 135 patients with severe systemic mastocytosis according to the blinded central document reading, $108(80 \%)$ fulfilled the criteria for WHO classification of indolent systemic mastocytosis. Hence, 27 patients (20\%) did not comply with the standard WHO classification but were still eligible for inclusion in the ITT population according to the non-standard protocol definition of indolent systemic mastocytosis based on histological criteria of typical mast cell infiltrates in a multifocal or diffuse pattern in skin or bone marrow biopsy (appendix p 21). Finally, although protocol amendments made during the study to improve the benefit-risk balance of the protocol are less than optimal, such changes did not bias the key findings (appendix p 5).

With regard to the mechanism of action, because the KIT Asp816Val mutation might not activate mast cells to release pro-inflammatory mediators-which is consistent with clinical observations that type and severity of symptoms are KIT Asp816Val-independentthe inactivity of masitinib against this target is not necessarily a limitation. ${ }^{1,5,6,25}$ The treatment effect is hypothesised to be a result of masitinib targeting wildtype mast cells, leading to a reduction in mast cell burden (an effect seen in long-term treatment of chronic myeloid leukaemia with the wild-type KIT-inhibitor imatinib; appendix $\mathrm{p} 17$ ), , 2,27 or by reducing activation of KIT Asp816Val mast cells. The latter proposed mechanism is mediated through dual inhibition of LYN and FYN, which contribute to modulation of mast cell 


\begin{tabular}{|c|c|c|c|c|c|c|c|c|c|}
\hline & \multicolumn{3}{|c|}{ Weeks 0-24 } & \multicolumn{3}{|c|}{ Extension period } & \multicolumn{3}{|c|}{ Overall study period } \\
\hline & $\begin{array}{l}\text { Masitinib } \\
(\mathrm{pm}=307)\end{array}$ & $\begin{array}{l}\text { Placebo } \\
(\mathrm{pm}=326)\end{array}$ & Difference & $\begin{array}{l}\text { Masitinib } \\
(\mathrm{pm}=1016)\end{array}$ & $\begin{array}{l}\text { Placebo } \\
(\mathrm{pm}=705)\end{array}$ & Difference & $\begin{array}{l}\text { Masitinib } \\
(\mathrm{pm}=1321)\end{array}$ & $\begin{array}{l}\text { Placebo } \\
(\mathrm{pm}=1031)\end{array}$ & Difference \\
\hline At least one adverse event & $22 \cdot 8$ & $19 \cdot 3$ & $3 \cdot 5$ & $3 \cdot 3$ & $3 \cdot 7$ & $-0 \cdot 4$ & $5 \cdot 3$ & $6 \cdot 1$ & -0.8 \\
\hline Death & 0.0 & $0 \cdot 3$ & $-0 \cdot 3$ & $0 \cdot 0$ & 0.0 & 0.0 & 0.0 & 0.1 & $-0 \cdot 1$ \\
\hline Non-fatal serious adverse event & 6.5 & $3 \cdot 7$ & $2 \cdot 8$ & 1.0 & $1 \cdot 1$ & -0.1 & $2 \cdot 1$ & 1.8 & $0 \cdot 3$ \\
\hline Severe adverse event & $11 \cdot 4$ & $6 \cdot 7$ & $4 \cdot 7$ & $1 \cdot 7$ & 1.4 & $0 \cdot 3$ & $3 \cdot 2$ & $2 \cdot 9$ & $0 \cdot 3$ \\
\hline $\begin{array}{l}\text { Adverse event leading to permanent } \\
\text { discontinuation (except death) }\end{array}$ & $5 \cdot 5$ & 1.2 & $4 \cdot 3$ & $0 \cdot 3$ & 0.0 & $0 \cdot 3$ & $1 \cdot 5$ & 0.4 & $1 \cdot 1$ \\
\hline $\begin{array}{l}\text { Adverse event leading to dose } \\
\text { reduction }\end{array}$ & 4.9 & $0 \cdot 3$ & $4 \cdot 6$ & $0 \cdot 3$ & 0.0 & $0 \cdot 3$ & 1.4 & 0.1 & $1 \cdot 3$ \\
\hline $\begin{array}{l}\text { Incidence in patient-months }(\mathrm{pm}) \text { is nur } \\
100 \mathrm{pm} \text {. Adverse events reported accorc }\end{array}$ & $\begin{array}{l}\text { r of patie } \\
\text { to any ca }\end{array}$ & $\begin{array}{l}\text { with at leas } \\
\text { ity. }\end{array}$ & e adverse & divided $b$ & sum of $e$ & e dura & months & quency is & lated per \\
\hline
\end{tabular}

degranulation in a KIT Asp816Val-independent manner.? The decrease we noted in mean tryptase levels in patients in the masitinib treatment arm is consistent with either of these effects-as are the individual decreases seen in most masitinib-treated patients ( 34 of $40,85 \%$; appendix pp 15-16). However, unknown factors could also contribute to these effects, as evidenced by the nonuniversal patient susceptibility to masitinib, with identification of predictive markers for patient treatment selection remaining a goal for future research.

Masitinib was associated with increased frequency of adverse events during the first 6 months of treatment compared with placebo, although no toxicities were lifethreatening, and over the long term ( $>1000$ patientmonths) the incidence of adverse events was similar between masitinib and placebo. Toxicities were predominantly gastrointestinal or skin events, consistent with the known adverse-event profile of masitinib, and which can be managed by dose reduction. Emerging evidence from the overall safety profile of masitinib shows that a substantial improvement in tolerance of masitinib occurs after the initial 12-week treatment period (unpublished data). These toxicities could be mitigated via implementation of a dose-escalation scheme-eg, initial dose of 3.0 or $4.5 \mathrm{mg} / \mathrm{kg}$ per day with increments of $1.5 \mathrm{mg} / \mathrm{kg}$ per day every 4 weeks depending on absence of toxicity until reaching the target dose of $6 \mathrm{mg} / \mathrm{kg}$ per day. Nevertheless, the safety profile of masitinib (including tolerance and toxicities) still compared favourably against that reported for interferon alfa, thalidomide, and cladribine-three drugs used in indolent mastocytosis. Treatment with interferon alfa has been associated with a variety of severe adverse events in almost every organ system, as well as with high levels of severe depression and severe cytopenia in patients with systemic mastocytosis. ${ }^{28,29}$ Use of thalidomide in patients with systemic mastocytosis is associated with severe peripheral neuropathy and severe myelosuppression..$^{30} \mathrm{~A}$ retrospective study ${ }^{31}$ of 68 patients showed frequent severe (grade 3-4) adverse events with use of cladribine in mastocytosis, including lymphopenia, neutropenia, and opportunistic infections, of which one was fatal.

Unlike aggressive forms of mastocytosis, indolent systemic mastocytosis-a rare condition with high, unmet medical need-has no registered or established standard treatment. Results from this study have shown a positive benefit-risk ratio for masitinib in severely symptomatic patients with indolent systemic mastocytosis, including the subvariant of smouldering systemic mastocytosis, as evidenced by a sustained response and long-term incidence of adverse events that was equivalent to placebo. Masitinib might therefore be an important new treatment option for these patients; moreover, these data suggest a possibility for effective longer-term management of this difficultto-treat disease.

\section{Contributors}

OH, OL, AM, LBA, J-PK, and PD contributed to study design. OL, MOC, CBL, CP, GG, EJ, MN, SB, SV, CG, GD, DC, SF, LL, SGL, KH, JA, RG, and PD contributed to data collection. $\mathrm{OH}, \mathrm{OL}, \mathrm{AM}$, and $\mathrm{CM}$ did the initial data interpretation. CM wrote the manuscript with major contributions from AM, OH, OL, and LBA. All authors contributed to final data interpretation and approved the final draft of the manuscript.

\section{Declaration of interests}

Masitinib is under clinical development by the study funder, AB Science. $\mathrm{AM}, \mathrm{CM}, \mathrm{KH}$, and JA are employees and shareholders of AB Science. $\mathrm{OH}$ is the President of the Scientific Committee of AB Science. PD, CA, and J-PK are consultants and shareholders of $\mathrm{AB}$ Science. All remaining authors have no competing interests.

\section{Acknowledgments}

We thank the patients enrolled in the study and their families. We also thank all of the investigators (appendix p 23) who contributed to the study. Financial support for medical editorial assistance (CM) was provided by AB Science.

\section{References}

1 Hermine O, Lortholary O, Leventhal PS, et al. Case-control cohort study of patients' perceptions of disability in mastocytosis. PLoS One 2008; 3: e2266.

2 Jennings S, Russell N, Jennings B, et al. The Mastocytosis Society survey on mast cell disorders: patient experiences and perceptions. J Allergy Clin Immunol Pract 2014; 2: 70-76.

3 Gleixner KV, Mayerhofer M, Cerny-Reiterer S, et al. KITD816V-independent oncogenic signaling in neoplastic cells in systemic mastocytosis: role of Lyn and Btk activation and disruption by dasatinib and bosutinib. Blood 2011; 118: 1885-98. 
4 Molderings GJ. The genetic basis of mast cell activation diseaselooking through a glass darkly. Crit Rev Oncol Hematol 2015; 93: 75-89.

5 Broesby-Olsen S, Kristensen T, Vestergaard H, et al. KIT D816V mutation burden does not correlate to clinical manifestations of indolent systemic mastocytosis. J Allergy Clin Immunol 2013; 132: 723-28.

6 Hoermann G, Gleixner KV, Dinu GE, et al. The KIT D816V allele burden predicts survival in patients with mastocytosis and correlates with the WHO type of the disease. Allergy 2014; 69: 810-13.

7 Dubreuil P, Letard S, Ciufolini M, et al. Masitinib (AB1010), a potent and selective tyrosine kinase inhibitor targeting KIT. PLoS One 2009; 4: e7258.

8 Gilfillan AM, Rivera J. The tyrosine kinase network regulating mast cell activation. Immunol Rev 2009; 228: 149-69.

9 Metcalfe DD. Mast cells and mastocytosis. Blood 2008; 112: 946-56.

10 Anastassiadis T, Deacon SW, Devarajan K, Ma H, Peterson JR Comprehensive assay of kinase catalytic activity reveals features of kinase inhibitor selectivity. Nat Biotechnol 2011; 11: 1039-45.

11 Davis MI, Hunt JP, Herrgard S et al. Comprehensive analysis of kinase inhibitor selectivity. Nat Biotechnol 2011; 29: 1046-51.

12 Paul C, Sans B, Suarez F, et al. Masitinib for the treatment of systemic and cutaneous mastocytosis with handicap: a phase $2 \mathrm{a}$ study. Am J Hematol 2010; 85: 921-25.

13 Valent P, Horny HP, Escribano L, et al. Diagnostic criteria and classification of mastocytosis: a consensus proposal. Leuk Res 2001; 25: 603-25.

14 Valent P, Horny H-P, Li CY. Mastocytosis. In: Jaffe ES, Harris NL, Stein H, Vardiman JW, eds. World Health Organization (WHO) Classification of Tumours. Lyon, France: IARC Press, 2001: 291-302.

15 Arock M, Akin C, Hermine O, Valent P. Current treatment options in patients with mastocytosis: status in 2015 and future perspectives. Eur J Haematol 2015; 94: 474-90.

16 American Psychiatric Association. Handbook of Psychiatric Measures. Washington, DC: American Psychiatric Association, 2000

17 Fisk JD, Ritvo PG, Ross L, Haase DA, Marrie TJ, Schlech WF. Measuring the functional impact of fatigue: initial validation of the fatigue impact scale. Clin Infect Dis 1994; 18: S79-83.

18 Committee for Medicinal Products for Human Use (CHMP). Guideline on clinical trials in small populations. CHMP/ EWP/83561/2005. London: European Medicines Agency, 2006.
19 Rochon, J. Application of GEE procedures for sample size calculations in repeated measures. Stat Med 1998; 17: 1643-58

20 Valent P, Akin C, Escribano L, et al. Standards and standardization in mastocytosis: consensus statements on diagnostics, treatment recommendations and response criteria. Eur J Clin Invest 2007; 37: 435-53.

21 Ballinger GA. Using generalized estimating equations for longitudinal data analysis. Organ Res Methods 2004; 7: 127-50.

22 Moura DS, Sultan S, Georgin-Lavialle S, et al. Depression in patients with mastocytosis: prevalence, features and effects of masitinib therapy. PLoS One 2011; 6: e26375.

23 Georgin-Lavialle S, Moura DS, Salvador A, et al. Mast cells' involvement in inflammation pathways linked to depression: evidence in mastocytosis. Mol Psychiatry 2016; 21: 1511-16.

24 Hermine O, Moussy A, Mansfield C, Dubreuil P. Masitinib for the treatment of severe systemic mastocytosis: pooled phase 2 study simulation of phase 3 population and response criteria. Proceedings of the EMBRN Meeting (21-23 October 2015, Marseille, France). http://www.ab-science.com/pdf/Hermine_2015_EMBRN_ Proceedings.pdf (accessed Nov 8, 2016).

25 Saleh R, Wedeh G, Herrmann H, et al. A new human mast cell line expressing a functional IgE receptor converts to tumorigenic growth by KIT D816V transfection. Blood 2014; 124: 111-20.

26 Parravicini V, Gadina M, Kovarova M, et al. Fyn kinase initiates complementary signals required for IgE-dependent mast cell degranulation. Nat Immunol 2002; 3: 741-48.

27 Cerny-Reiterer S, Rabenhorst A, Stefanzl G, et al. Long-term treatment with imatinib results in profound mast cell deficiency in $\mathrm{Ph}+$ chronic myeloid leukemia. Oncotarget 2015; 6: 3071-84.

28 Sleijfer S, Bannink M, Van Gool AR, Kruit WH, Stoter G. Side effects of interferon-alpha therapy. Pharm World Sci 2005; 27: 423-31.

29 Casassus P, Caillat-Vigneron N, Martin A, et al. Treatment of adult systemic mastocytosis with interferon-alpha: results of a multicentre phase II trial on 20 patients. Br J Haematol 2002; 119: 1090-97.

30 Gruson B, Lortholary O, Canioni D, et al. Thalidomide in systemic mastocytosis: results from an open-label, multicentre, phase II study. Br J Haematol 2013; 161: 434-42.

31 Barete S, Lortholary O, Damaj G, et al. Long-term efficacy and safety of cladribine (2-CdA) in adult patients with mastocytosis. Blood 2015; 126: 1009-16. 